\title{
Propensity matching cannot substitute for randomization in albumin studies
}

\author{
Christian J Wiedermann ${ }^{1 *}$ and Wolfgang Wiedermann ${ }^{2}$ \\ See related article by Frenette et al., http://ccforum.com/content/18/6/602
}

Large randomized trials such as the Albumin Italian Outcome Sepsis (ALBIOS) trial involving 1,818 patients with severe sepsis have revealed no evidence of acute kidney injury (AKI) attributable to albumin infusion [1]. Such results are difficult to reconcile with an association between albumin and AKI in the retrospective study by Frenette and colleagues of 984 cardiac surgery patients receiving $6 \%$ hydroxyethyl starch $130 / 0.4,10 \%$ pentastarch, $5 \%$ albumin and/or $25 \%$ albumin in unspecified combinations [2]. Those investigators criticize the ALBIOS trial for a lack of data on timing of AKI in relation to albumin infusion, but that is also among the shortcomings of their own study. Baseline data are not stratified by colloid group, so imbalances cannot be assessed. The doseresponse analysis is univariate only. The propensity matching did not include known independent risk factors for AKI such as preoperative hypoalbuminemia [3] and cardiac catheterization [4]. Furthermore, the matching failed to achieve satisfactory balance, since there remained a significant difference in concomitant pentastarch dose.

Frenette and colleagues misinterpret a study on the robustness of propensity scores [5] as suggesting that their study may have underestimated AKI risk associated with albumin infusion. The study showed that mortality was decreased by albumin in randomized trials of critically ill patients with an odds ratio of 0.82 and a $95 \%$ confidence interval of 0.67 to 1.00 , but mortality was increased with propensity score matching of observational study data (odds ratio, 2.02; confidence interval, 1.43 to 2.84). The difference was significant (ratio of odds ratio, 0.43; confidence interval, 0.29 to 0.63 ). Thus, propensity scores evidently cannot overcome the tendency to use albumin as salvage treatment in sicker patients.

\section{Authors' response}

\section{Josée Bouchard, Anne Julie Frenette, Stéphan Troyanov and David R Williamson}

We agree with Wiedermann and Wiedermann that propensity scores will never replace randomized controlled trials. However, the study design of the ALBIOS trial differed from our study on several points [1]. In the ALBIOS trial, albumin was administered to patients with hypoalbuminemia and severe sepsis or shock according to albumin levels, and not according to the clinical context. In addition, the mean daily dose of albumin administered was substantially lower in this randomized trial $(0.4 \mathrm{mg} / \mathrm{kg} /$ day over the first 7 days vs $0.9 \mathrm{mg} / \mathrm{kg} /$ day in our study) and the period of administration was much longer than our study ( 28 days vs 36 hours in our study) $[1,2]$. The timing of AKI in relation to albumin

\footnotetext{
* Correspondence: christian.wiedermann@asbz.it

'Department of Internal Medicine, Central Hospital of Bolzano/Bozen, Teaching Hospital of the Medical University of Innsbruck, Lorenz-Böhler Street 5, 39100 Bolzano/Bozen (BZ), Italy

Full list of author information is available at the end of the article
}

administration was not detailed in the ALBIOS trial, while we summarized these results in a figure and in the text.

Regarding our study, the unbalanced results from the matching of our propensity score would have biased the results toward a decreased AKI rate in the albumin group. In addition, the percentage of patients undergoing cardiac catheterization was similar between the no albumin and albumin groups in the propensity score (27.0\% vs $25.5 \%, P=0.79)$. As mentioned, our study does not provide a definitive answer on the use of albumin in patients undergoing cardiac surgery. Future studies should be performed on the use of albumin in surgical populations.

\section{Abbreviations}

AKI: Acute kidney injury; ALBIOS: Albumin Italian Outcome Sepsis.
C Biomed Central

(c) 2015 Wiedermann and Wiedermann; licensee BioMed Central. This is an Open Access article distributed under the terms of the Creative Commons Attribution License (http://creativecommons.org/licenses/by/4.0), which permits unrestricted use, distribution, and reproduction in any medium, provided the original work is properly credited. The Creative Commons Public Domain Dedication waiver (http://creativecommons.org/publicdomain/zero/1.0/) applies to the data made available in this article, unless otherwise stated. 


\section{Competing interests}

CJW has received fees for speaking and travel reimbursements from manufacturers of plasma-derived therapies (CSL Behring, Kedrion, Baxter). WW declares that he has no competing interests.

\section{Authors' contributions}

CJW developed the concept for the letter and drafted the manuscript. Both authors revised the manuscript and read and approved the final version.

\section{Acknowledgements}

This letter is solely the work product of the authors. No other individual or organization made any substantial contribution to the creation and revision of the letter.

\section{Author details}

'Department of Internal Medicine, Central Hospital of Bolzano/Bozen, Teaching Hospital of the Medical University of Innsbruck, Lorenz-Böhler Street 5, 39100 Bolzano/Bozen (BZ), Italy. ${ }^{2}$ Department of Psychology, Unit of Quantitative Methods, University of Vienna, Liebiggasse 5, 1010 Vienna, Austria.

\section{Published online: 28 April 2015}

\section{References}

1. Caironi P, Tognoni G, Masson S, Fumagalli R, Pesenti A, Romero M, et al. Albumin replacement in patients with severe sepsis or septic shock. N Engl J Med. 2014;370:1412-21.

2. Frenette AJ, Bouchard J, Bernier P, Charbonneau A, Nguyen LT, Rioux J-P, et al. Albumin administration is associated with acute kidney injury in cardiac surgery: a propensity score analysis. Crit Care. 2014;18:602.

3. Lee EH, Baek SH, Chin JH, Choi DK, Son HJ, Kim WJ, et al. Preoperative hypoalbuminemia is a major risk factor for acute kidney injury following off-pump coronary artery bypass surgery. Intensive Care Med. 2012;38:1478-86.

4. Del Duca D, labal S, Rahme E, Goldberg P, de Varennes B. Renal failure after cardiac surgery: timing of cardiac catheterization and other perioperative risk factors. Ann Thorac Surg. 2007:84:1264-71.

5. Zhang $\mathrm{Z}, \mathrm{Ni} \mathrm{H}, \mathrm{Xu} \mathrm{X}$. Observational studies using propensity score analysis underestimated the effect sizes in critical care medicine. J Clin Epidemiol. 2014;67:932-9. 